\title{
Naringenin promotes SDF-1/CXCR4 signaling pathway in BMSCs osteogenic differentiation
}

\author{
Yipei Wang ${ }^{1,2}$, Shulin Bai1 ${ }^{1,2}$, Qian Cheng ${ }^{1,2}$, Yang Zeng ${ }^{1,2}$, Xiaomei Xut ${ }^{1,2}$, Guangzhao Guan ${ }^{3}$ \\ ${ }^{1}$ Department of Orthodontics, The Affiliated Stomatology Hospital of Southwest Medical University, \\ Luzhou, Sichuan 646000, P.R. China \\ ${ }^{2}$ Oral and Maxillofacial Reconstruction and Regeneration Laboratory, Southwest Medical University, \\ Luzhou, P.R. China \\ ${ }^{3}$ Department of Oral Diagnostic and Surgical Sciences, Faculty of Dentistry, University of Otago, \\ Dunedin, New Zealand
}

\begin{abstract}
Introduction. Naringenin, a dihydro-flavonoid compound that shows chemotactic activity, may have a good application prospect in repairing bone tissue, but its specific mechanism in bone regeneration, especially the osteogenic differentiation of stem cells, needs for a further study. The aim of this study was to investigate the effect of naringenin on the osteogenic differentiation and its roles in the C-X-C chemokine receptor type 4/stromal cell-derived factor 1 (SDF-1/CXCR4) signal pathway of bone marrow-derived mesenchymal stem cells (BMSCs). Material and methods. BMSCs were harvested from the femurs and tibias of 4-to-6-week-old male Sprague-Dawley rats. Cell Counting kit-8 assay was used to determine cytotoxicity of naringenin. Alkaline phosphatase (ALP) activity was measured in cell's precipitates and alizarin-red staining was performed to determine the osteogenic differentiation capacity of the BMSCs. Real-time polymerase chain reaction, enzyme-linked immunosorbent assay and western blotting were adopted to determine the expression of genes and proteins.

Results. The cellular morphology was spindle-shaped, and arranged in radial and whorled patterns. The flow cytometric analysis have confirmed the presence of characteristic surface proteins in the harvested BMSCs. Different concentrations $(0-200 \mu \mathrm{g} / \mathrm{ml})$ of naringenin have no influence on the viability and proliferation rate of the BMSCs. The highest ALP activity was found at culture day 7 and 9 when the concentration of naringenin was 75 and $100 \mu \mathrm{g} / \mathrm{ml}$. Positive red or dark red stained cells with mineralized nodules can be observed on day 14. The expression of ALP, Runt-related transcription factor 2, CXCR4 and SDF-1 $\alpha$ at the gene and protein levels in naringenin-treated cells were significantly higher than those in the control cells. Moreover, AMD3100, an inhibitor of CXCR4, suppressed the expression of the studied genes and proteins.

Conclusions. Naringenin does not show toxic effect on BMSCs. Naringenin promotes the expression of the SDF-1 $\alpha$ gene and protein via the SDF-1/CXCR4 signaling pathway. A better understanding of the mechanisms of naringenin action would be helpful for developing specific therapeutic strategies to improve bone regeneration after injuries. (Folia Histochemica et Cytobiologica 2021, Vol. 59, No. 1, 66-73)
\end{abstract}

Key words: bone marrow-derived mesenchymal stem cells; cell viability; naringenin; alizarin red; SDF-1; CXCR4; RT-qPCR

Correspondence address: Xiaomei $\mathrm{Xu}$,

Department of Orthodontics, The Affiliated Stomatology

Hospital of Southwest Medical University, 2 Jiangyang South

Road, Luzhou, Sichuan 646000, P.R. China

e-mail: xuxiaomei@hotmail.com

\section{Introduction}

Bone has a remarkable capacity of spontaneous repairing and regeneration. The process of spontaneous bone regeneration using bone marrow stroma-derived

This article is available in open access under Creative Common Attribution-Non-Commercial-No Derivatives 4.0 International (CC BY-NC-ND 4.0) license, allowing to download articles and share them with others as long as they credit the authors and the publisher, but without permission to change them in any way or use them commercially. (c) Polish Society for Histochemistry and Cytochemistry 
stem cells (BMSCs) holds a great potential, which would greatly affect the human health by a way of providing the possibility to repair tissues injury [1,2]. BMSCs, the first important components and regulators to be found in bone marrow, produce hematopoietic-supporting stromal cells and form the hematopoietic niches for hematopoietic stem cells $[3,4]$.

Chemokines are the important regulators of BMSCs. The SDF-1/CXCR4 signaling pathway appears important in maintaining proliferation and survival of BMSCs by improving cellular migration, remodeling and survival in response to stress $[5,6]$. In bone remodeling, the SDF-1/CXCR4 signaling pathway regulates the osteogenic differentiation of the BMSCs, the expression of osteogenic protein such as ALP, OCN and OSX and the formation of osteoblasts. It is also closely related to bone morphogenic protein 2 signaling [7]. CXCR4-knockout mice showed the reduction in bone formation and the osteogenic differentiation of BMSCs $[8,9]$. The osteogenic effect of SDF-1/ CXCR4 signaling can be further enhanced by other molecules such as hypoxia-inducible factor (HIF) family members, resulting in strong synergistic effects on the bone remodeling and regeneration [10].

Although chemokines can be synthesized chemically, the expensive cost limits their clinical application. Naringenin (4',5-7 trihydroxydihydroflavone, Fig. 1), widely present in fruits such as orange, pomelo, and drynaria, is a dihydro-flavonoid compound that shows chemotactic activity [11] and other biological effects such as anti-inflammatory, antioxidative, anticancer and antiadipogenic activities [12-15], and is also shown to have anti-osteoporotic and anti-osteolytic effects [16]. Several experiments have shown that naringenin could reduce the osteolytic activities of osteoclasts, and improve the osteogenic differentiation and mineralization effects of the osteoblasts in osteolysis-related diseases [16-20]. The aim of the study was to investigate the effect

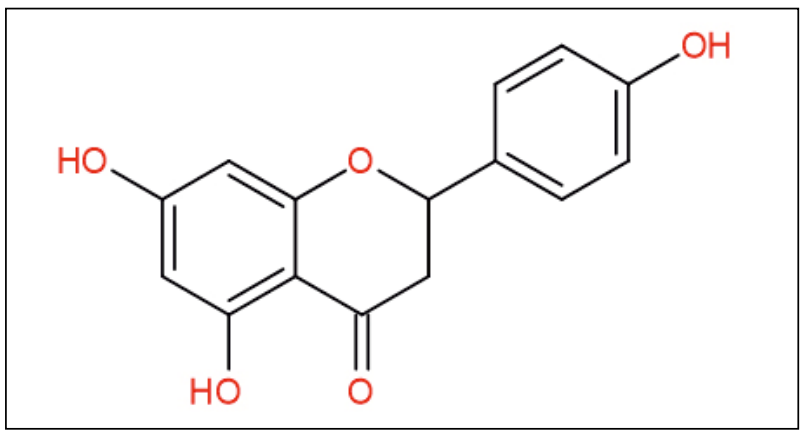

Figure 1. The chemical structure of naringenin [38]. of naringenin on the osteogenic differentiation of BMSCs and its role in the SDF-1/CXCR4 signaling pathway.

\section{Materials and methods}

Isolation, culture and identification of BMSCs. All experiments with the use of animals were conducted under the Guidelines for Animal Experimentation and were approved by the Ethics Committee of Southwest Medical University (Luzhou, China, Certificate No.201812-25). BMSCs were harvested from the femurs and tibias of 4-to-6-week-old rats as previously reported [21]. Briefly, rats were killed with chloral hydrate, and the tibia and femur were obtained. The bilateral epiphyseal end of the bone was cut by scissors, and then the D-Hank's solution (Solarbio, Beijing, China) containing $10 \%$ fetal bovine serum (FBS, Sijiqing, Hangzhou, China) was extracted by syringe to wash the bone marrow cavity.

Cells were incubated in F-12 medium (Gibco, Grand Island, NY, USA) with $10 \%$ FBS and $1 \%$ antibiotics $(100 \mathrm{U} / \mathrm{mL}$ penicillin and $100 \mu \mathrm{g} / \mathrm{mL}$ streptomycin, HyClone, South Logan, UT, USA) in a $37^{\circ} \mathrm{C}$ humidified atmosphere with $5 \% \mathrm{CO}_{2}$ in air. When the monolayer reached approximately $80 \%$ confluence, cells were passaged. Then, the passages 2 through 3 (P2-P3) cells were used for this study.

After the second passage, cells were characterized by flow cytometry for the expression of the BMSCs surface antigens, such as CD29, CD34, CD45 and CD90. After that the cells cultured with lipogenic induction medium ( $\alpha$-MEM, FBS, penicillin-streptomycin solution, IBMX, dexamethasone, indometacin, insulin (all from SigmaAldrich, St. Louis, MO, USA) were stained with Oil Red $\mathrm{O}$ at day 12 to identify lipid vacuoles, and the cells cultured with osteogenic induction medium ( $\alpha$-MEM, FBS, penicillin-streptomycin solution, sodium $\beta$-glycerophosphate, vitamin $\mathrm{C}$ and dexamethasone; all from SigmaAldrich) were stained with Alizarin Red on day 14 to characterize osteogenic activities.

Cell Counting kit-8 (CCK-8) viability assay. To detect the potential cytotoxicity of naringenin on the BMSCs, the CCK-8 assay was performed [22]. A set of classic osteogenesis inducers group was used as positive control. The culture medium was used as negative control. BMSCs were incubated with different naringenin concentrations of $0,6.25$, $12.5,25,50,75,100,200 \mu \mathrm{g} / \mathrm{ml}$. Rat BMSCs at passage 2 were transferred to 96 -well plates at $1 \times 10^{4} /$ well density. When the cells reached $80 \%$ confluence, different reagents were added by categorization. On day 2, $10 \mu \mathrm{l}$ of CCK-8 reagents (Dojindo-Kumamoto, Japan) were added to each well. The optical density (OD) at $450 \mathrm{~nm}$ wavelength was measured by a Varioskan Flash enzyme-labeled instrument (Thermo Fisher, Waltham, MA, USA). Cell viability was then calculated based on OD values. 
Quantification of alkaline phosphatase (ALP) activity. ALP quantification assay was performed to determine the osteogenic differentiation capacity of BMSCs in the control and experimental groups on day 3, 5, 7 and 9 [23]. BMSCs were placed in 24-well plates at a cell density of $5 \times 10^{4} /$ /well. After being cultured for the indicated time, the cells were centrifuged in a centrifuge tube and lysed by $0.1 \%$ TritonX-100 (Beyotime, Shanghai, China). After the cells were sufficiently lysed, the cells were centrifuged at 13,000 rpm for $5 \mathrm{~min}$. Then the supernatant was proceeded according to the manufacturer's instruction of ALP activity assay kit (Nanjing Jiancheng Bioengineering Institute, Nanjing, China). The OD value was measured at the $520 \mathrm{~nm}$ wavelength.

Alizarin red staining. BMSCs were placed in $35 \mathrm{~mm}$ Petri dishes at a cell density of $5 \times 10^{5} /$ well. When the cells reached $80 \%$ confluence, the culture medium was transferred to the control groups, $100 \mu \mathrm{g} / \mathrm{ml}$ naringenin group, $100 \mu \mathrm{g} / \mathrm{ml}$ naringenin + AMD3100 antagonist group and classical osteogenic induction group. 14 days later, cells were stained with alizarin red dye (Sangon, Shanghai, China).

Real-time polymerase chain reaction (RT-qPCR). Induction cells in each group were digested with trypsin and centrifuged, and the precipitates were collected on day 7. The total RNA was extracted by Trizol reagent (Ambion, Austin, TX, USA) and converted to cDNA (TAKARA, Mountain View, CA, USA). Real-time RT-PCR was performed using a SYBR Green PCR Kit (Kapa Biosystems, Wilmington, MA, USA). The primer sequences were shown in Table 1.

Enzyme-linked immunosorbent assay (ELISA). The concentrations of SDF- $1 \alpha$ and CXCR4 proteins in the cell supernatants were measured by ELISA (Bioswamp, Wuhan, China) according to the manufacturer's instructions on day 1, 3, 5 and 7 .

Western blotting. The cells in each group were lysed with RIPA lysis buffer (Beyotime, Shanghai, China), after induced

Table 1. The primers sequences used for RT-qPCR

\begin{tabular}{|l|c|}
\hline Gene & Primer Sequence \\
\hline SDF- $1 \alpha-\mathrm{F}$ & TTGTCTCAACCCTGAAGCCC \\
\hline SDF- $1 \alpha-\mathrm{R}$ & TGCCCGTTGAGGTACAGGAG \\
\hline CXCR4-F & CCTCCTCCTGACTATCCCTGA \\
\hline CXCR4-R & CGAACTCACATCCTTGCTTG \\
\hline ALP-F & CTACCACTCGGGTGAACCA \\
\hline ALP-R & AGCTGATATGCGATGTCCTT \\
\hline Runx2-F & AACTTCCTGTGCTCCGTGCT \\
\hline Runx2-R & TCGTTGAACCTGGCTACTTGG \\
\hline GAPDH-F & CAAGTTCAACGGCACAG \\
\hline GAPDH-R & CCAGTAGACTCCACGACAT \\
\hline
\end{tabular}

for 7 days to obtain the total protein. Then, the BCA protein test kit was used to measure the total protein concentration. Equal amounts of cell extracts $(20 \mu \mathrm{g} /$ lane $)$ separated by $10 \%$ SDS-PAGE and proteins were transferred to PVDF membranes. After being sealed with 5\% skim milk overnight at $4^{\circ} \mathrm{C}$, the membranes were incubated with primary antibodies overnight at room temperature. The antibodies used were as following: rabbit anti-Alkaline Phosphatase (1: 1000, Abcam, Cambridge, MA, USA), mouse anti-Runt-related transcription factor 2 (RUNX2) (1: 1000, Abcam), rabbit anti-SDF-1/ /CXCL12 (1: 1000, CST, Danvers, MA, USA), rabbit anti-CXCR4 polyclonal antibody (1: 1000, Invitrogen, Carlsbad, CA, USA), rabbit anti-GAPDH (1:1000, Bioswamp). Horseradish peroxidase (HRP)-goat anti-rabbit IgG (1: 20000, Bioswamp) and HRP-rabbit anti-mouse IgG antibody (1: 20000, Bioswamp) were used as secondary antibodies for one hour at room temperature. We used glyceraldehyde 3-phosphate dehydrogenase as the internal control.

Statistical analysis. All the experimental data were expressed as mean \pm standard deviation (SD) from three independent experiments. Analysis of variance (ANOVA) is used for data analysis by the SPSS software (SPSS Inc., Chicago, IL, USA), $p$ values $<0.05$ were defined as statistically significant.

\section{Results}

\section{Culture and identification of BMSCs}

The cells appeared in sphere shape during the first two days. At day 6, the cells were spindle-shaped and bipolar, occasionally multipolar (Fig. 2A). After passage of the cells, cellular morphology became uniform, spindle-shaped, and arranged in radial and whorled patterns (Fig. 2B).

The phenotypic cells of BMSCs were identified by flow cytometry. BMSC-associated markers such as CD29 (99.08\%) and CD90 (96.06\%) were positive. The cells lacked the expression of hematopoietic markers (CD45 and CD34) (Fig. 2C). In the cell cycle assay, G1 cells accounted for $87.91 \%$ of all cells, and S-phase cells fraction was $8.22 \%$, given the ratio G2/ /G1 equaled to 1.90. This indicated that most of the cells were at rest, which was one of the characteristics of stem cells (Fig. 2D).

At day 14, the alizarin-red positively stained red or dark-red cells with mineralized nodules can be observed (Fig. 2E). At day 12, large lipid vacuoles stained with Oil Red $\mathrm{O}$ were observed in the BMSCs cultures (Fig. 2F). Thus, after 2 weeks of culture BMSCs showed both osteogenic and adipogenic differentiation potential. Experimental results showed that the cells obtained are BMSCs, which are of high purity, and meet the requirements of subsequent experiments. 


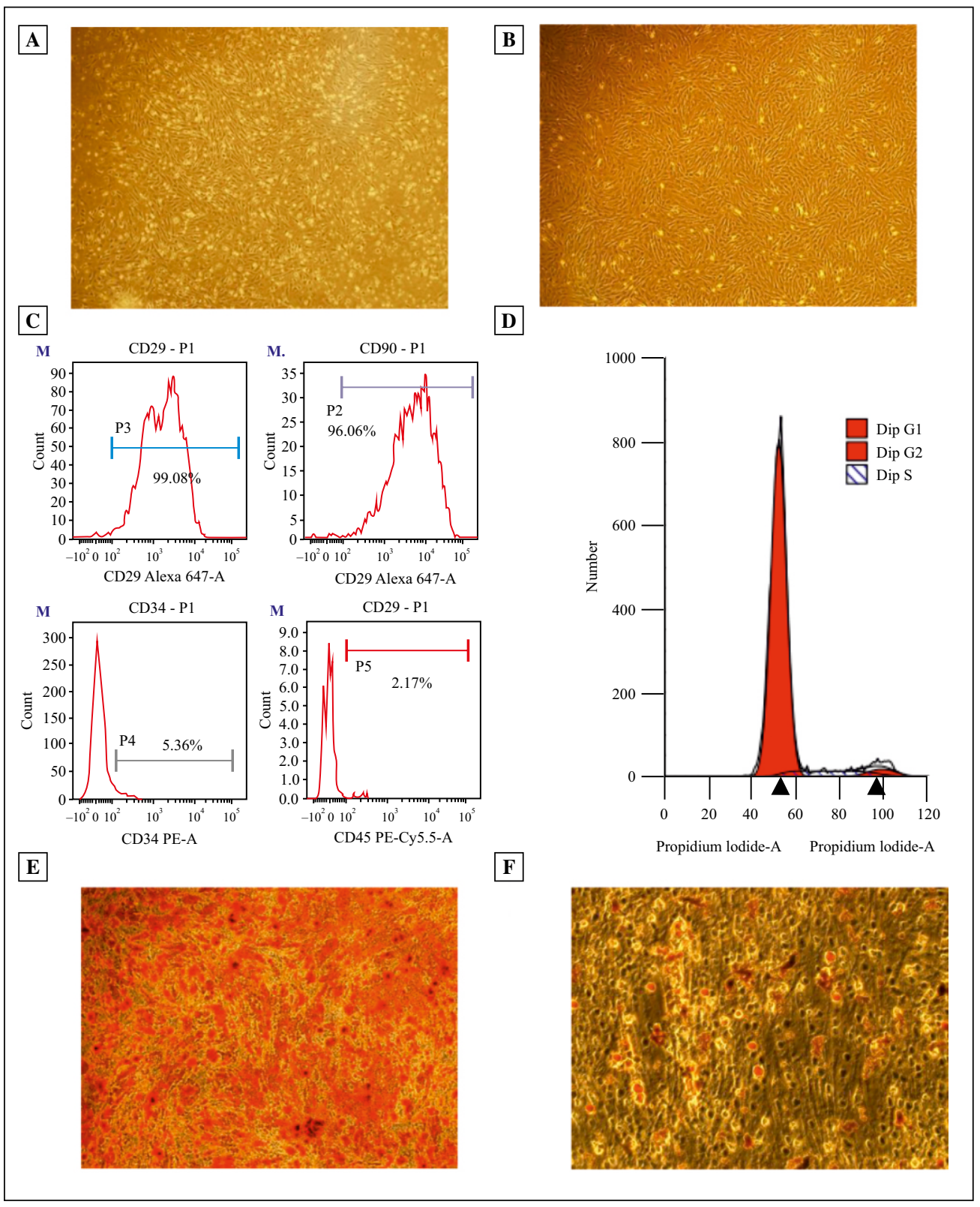

Figure 2. Culture, identification and characterization of rat BMSCs. A. BMSCs morphology on day $6(40 \times)$. B. The morphology of the cells became uniform, spindle-shaped, with well-defined cytoplasm after passage $2(40 \times)$. C. Flow cytometry results showed that the cells were positive for MSCs specific markers (CD29, CD90), and negative for hematopoietic markers (CD34, CD45). D. In the cell cycle assay by flow cytometry, G1 cells accounted for $87.91 \%$, and S-phase cells fraction was $8.22 \%$, given the ratio $\mathrm{G} 2 / \mathrm{G} 1$ equaled to 1.90 . E. Alizarin Red staining was used to demonstrate mineralized nodules in BMSCs cultured with osteogenic induction fluid on day $14(40 \times)$. F. Large lipid vacuoles were observed in BMSCs cultured with lipogenic induction medium at day 12 via Oil Red O assay $(100 \times)$.

Cell viability and osteogenic differentiation of BMSCs To explore the possible naringenin toxicity, the cellular viability was quantified for different concentrations of naringenin. In the experimental groups, no significant difference in cell viability was found between $6.25-200 \mu \mathrm{g} / \mathrm{ml}$ at day 2 (Fig. 3A). Thus, different concentrations of naringenin did not influence the viability and proliferation rate of the BMSCs.
Within the experimental groups, the trend of each group at different time points was the same, and it showed a time-dependent increase on days 3, 5, 7 and 9. The ALP activities in cell's precipitates of $75,100 \mu \mathrm{g} / \mathrm{ml}$ naringenin group were the highest at each time points, and the difference between the two groups was not statistically significant (Fig. 3B). 


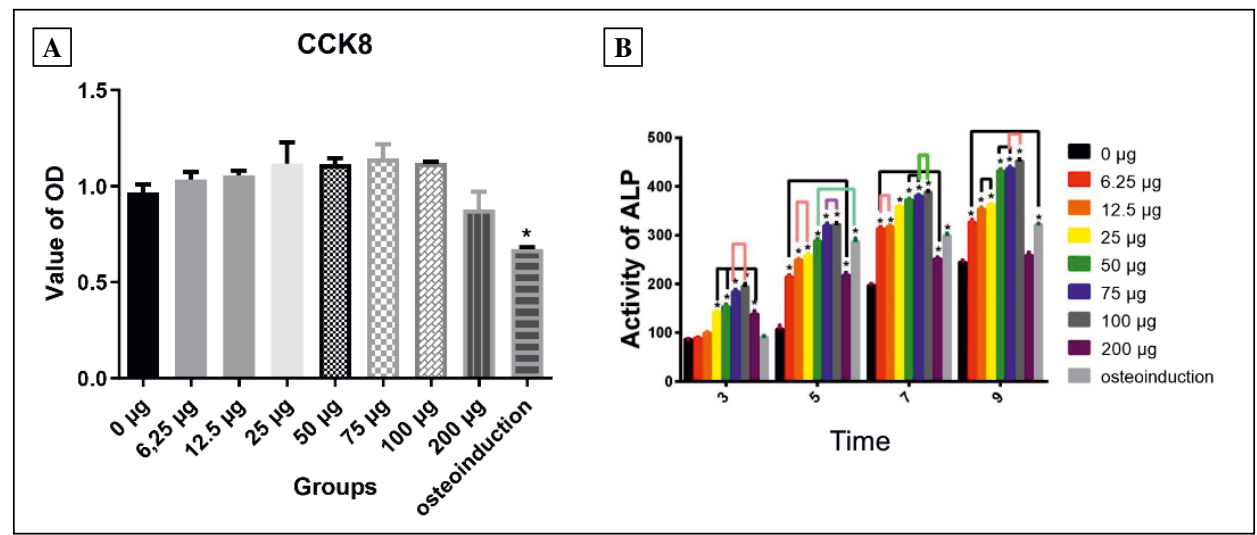

Figure 3. Cell viability and osteogenic differentiation of BMSCs. A. Different concentrations of naringenin have no influence on the viability and proliferation rate of the BMSCs as measured by CCK- 8 assay. B. High ALP activity was found at the concentration of 75 and $100 \mu \mathrm{g} / \mathrm{ml}$ of naringenin on days 3, 5, 7, and 9. Results are means \pm standard deviation (SD), and similar data were obtained from three independent experiments $(\mathrm{n}=3)$. The activity of ALP was measured in arbitrary units as described in Methods. * $p<0.05 v s$. the culture medium group (one-way ANOVA followed by Tukey's test).

\section{The role of naringenin in the SDF-1/CXCR4 signaling pathway}

To explore the effects of naringenin on the SDF-1 /CXCR4 signaling pathway, CXCR4 antagonist AMD3100wasadded to BMSCsincubated with $100 \mu \mathrm{g} / \mathrm{ml}$ naringenin. More mineralization nodules were noted in $100 \mu \mathrm{g} / \mathrm{ml}$ naringenin group when compared with the classic osteogenic induction and culture medium groups (negative control). However, the alizarin red staining was suppressed in AMD3100 $+100 \mu \mathrm{g} / \mathrm{ml}$ naringenin group when compared with $100 \mu \mathrm{g} / \mathrm{ml}$ naringenin and classic osteogenic induction groups (Fig. 4A).

The expression of ALP, RUNX2, CXCR 4 and SDF- $1 \alpha$ genes in cells incubated with naringenin, measured by RT-qPCR, were significantly higher than those in the cells of negative control. However, after adding AMD3100 to naringenin-treated cells, the expression of these genes were significantly suppressed (Fig. $4 \mathrm{~B})$. Similar results of SDF- $1 \alpha$ and CXCR 4 proteins by ELISA were found at different time lines, such as those on day 1, 3, 5, and 7 (Fig. 4C, D). The results of the expression of ALP, RUNX2, CXCR4 and SDF- $1 \alpha$ proteins measured by western blot were similar to the expression of their respective genes (Fig. 4E).

\section{Discussion}

Naringenin has many pharmacological activities, and has become one of the flavonoids concerned by scientists in many areas of biological and biomedical studies. With the further study of naringenin, it is found that naringenin has obvious effect on bone defect repair. Kaczmarczyk-Sedlak et al. [16] found that adding naringenin to the diet of Wistar rats could improve the symptoms of osteoporosis and the morphological manifestations of bone tissue after ovarian removal showing that naringenin has obvious anti-osteoporosis and anti-osteolysis effects in bone tissue repair. In addition, naringenin, the aglycone of naringin, is the most common form in serum after oral administration of naringin because naringin can be metabolized into absorbable naringenin by intestinal flora [24]. It is also reported that naringenin exhibited higher antioxidant capacity and hydroxyl and superoxide radical scavenger efficiency than naringin [12]. SDF-1/CXCR4 signaling pathway plays an important role in preserving the bone marrow stem cells in bone marrow and regulating the release of stem cells from bone marrow to peripheral circulation [25]. CXCR4 signals the stem cells migration during fracture healing. It participates in bone repair and regeneration by inducing cellular migration and regulating differentiation [9]. SDF-1 is induced in the periosteum of injured bone, and it promotes endochondral bone repair and regeneration by recruiting BMSCs to the site of injury [26, 27]. However, the role of the SDF-1/ /CXCR4 signaling pathway in osteogenic differentiation induced by naringenin is unknown.

It was previously shown that the highest ALP activity was found after day 8 in the naringenin group, which was confirmed by our results [28]. Cellular viability assay showed no significant toxicity among the experimental groups at concentration between $0 \sim 200 \mu \mathrm{g} / \mathrm{ml}$. Guo-Yong Y et al. [29] reported that treatment at the optimal concentration $(50 \mu \mathrm{g} / \mathrm{mL})$ for rat BMSCs increased mRNA levels of osteogenic genes (ALP, BSP, and cbfa1). In our study, the highest ALP activity value was found at day 7 and 9 at the naringenin concentration of 75 and $100 \mu \mathrm{g} / \mathrm{ml}$. Therefore, we chose $100 \mu \mathrm{g} / \mathrm{ml}$ over 7 days for our subsequent experiments. 


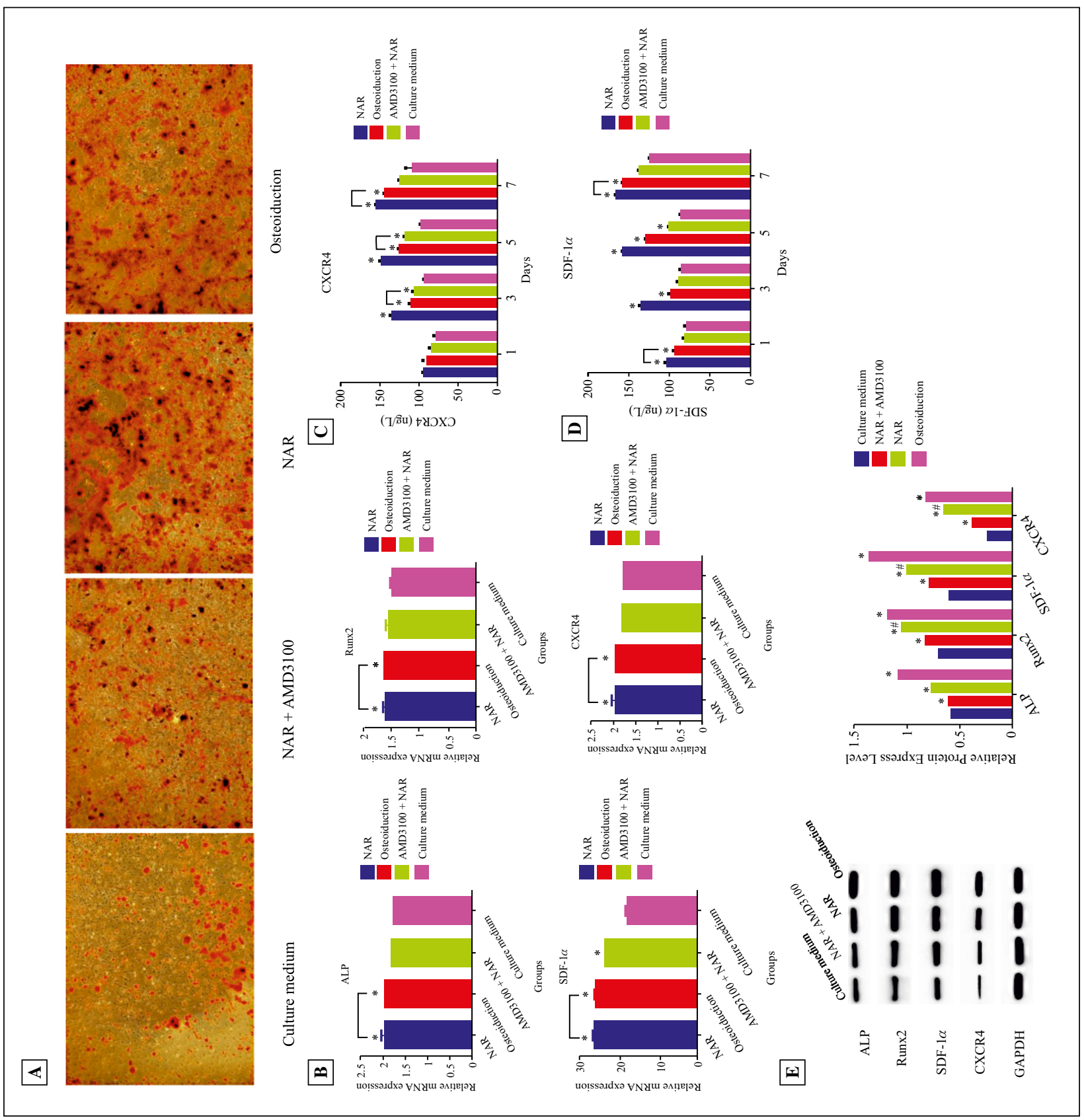

Figure 4. The role of naringenin in the SDF-1/CXCR4 signal pathway. A. Each group was stained with Alizarin Red on day 14. $(40 \times)$. B. The expression of osteogenic genes in naringenin groups was upregulated at the mRNA level as assessed by RT-qPCR when compared with culture medium group $(\mathrm{p}<0.05)$. C. The expression of CXCR4 protein in BMSCs induced by naringenin was higher than the culture medium group as measured by ELISA $(\mathrm{p}<0.05)$. D. The expression of SDF- $1 \alpha$ protein measured by ELISA in supernatants of naringenin-induced BMSCs was suppressed as compared with culture medium group. E. Suppression of ALP, RUNX2, SDF-1 $\alpha$, and CXCR4 proteins in BMSCs induced by naringenin were detected by Western blotting. Results are means \pm standard deviation $(\mathrm{SD}), \mathrm{n}=3 ;{ }^{*} p<0.05 v$ s. culture medium group, ${ }^{*} p<0.05 v s$. NAR + AMD3100 group. Abbreviations: NAR — naringenin; AMD3100, an antagonist of SDF-1/CXCR4 signaling.

This study further examined the role of SDF-1/ /CXCR4 signaling pathway in the BMSCs osteogenic differentiation by using the CXCR4 antagonist AMD3100. CXCR4 antagonists include AMD3100 and T140 [30, 31]. AMD3100 inhibits the binding of CXCR4 to SDF-1, and blocks signal transduction along the SDF-1/CXCR4 axis.

In this study, the mineralized nodules were significantly decreased after adding AMD3100 to BMSCs treated with naringenin, when compared with the 
osteogenic induction group and naringenin group. It suggested that AMD3100 might suppress the effect of naringenin in osteogenic differentiation by blocking the SDF-1/CXCR4 signaling pathway in BMSCs. The measurements of the expression of CXCR 4 and SDF- $1 \alpha$ genes and proteins showed that it increased over the induction time in the experimental groups. Naringenin promoted the expression the SDF- $1 \alpha$ gene and synthesis of protein in the early stage of osteogenic differentiation in BMSCs. Study has showed that the SDF-1 was upregulated at the fracture site and ischemic site after femoral bone transplantation in a mice model [32]. Kawakami et al. [5] found that bone healing was delayed by decreasing the callus formation in CXCR4-deficient mice. An in vitro study showed that overexpression of SDF- $1 \alpha$ in human BMSCs might promote osteogenic differentiation and osteoblastic angiogenesis by upregulating the osteogenic-related proteins, such as ALP, RUNX2, osteocalcin, and p-Smadl/5, and angiogenesis-related protein such as CD31 [33]. In the present study, naringenin regulated the SDF-1/CXCR4 signaling pathway by increasing the expression of SDF-1, and increased osteogenic differentiation of BMSCs. SDF-1 and CXCR4 activate a series of downstream signaling pathways, including extracellular signal-regulated kinases (ERK) 1/2 [34] and phosphoinositide 3-kinases/protein kinase B (PI3K/AKT) [35]. Naringin was shown to enhance osteogenic differentiation by activating ERK signaling pathway in human BMSCs [36] and PI3K/AKT signaling pathway in osteoblasts [37]. Naringenin might act on the same pathway and regulate the downstream pathways (ERK1/2 and PI3K/AKT) to induce osteogenesis. In addition, it was found that naringenin exerted its protective effects by inhibiting osteoclastogenesis and osteoclast bone resorption $[19,20]$. Therefore, naringenin might have therapeutic potentials for a range of bone diseases such as osteoporosis, repairing of bone defects, alveolar bone resorption and other.

In summary, we found that naringenin does not have toxic effects on BMSCs. Naringenin promoted the expression of the ALP, RUNX2, SDF- $1 \alpha$ and CXCR4 genes and proteins via the SDF-1/CXCR4 signaling pathway. A better understanding of the molecular mechanisms of naringenin activity will be beneficial in developing specific therapeutic strategies to improve bone repair after injuries.

\section{Conflict of interests}

The authors declare that they have no conflict of interest.

\section{References}

1. Hokugo A, Saito T, Li A, et al. Stimulation of bone regeneration following the controlled release of water-insoluble oxysterol from biodegradable hydrogel. Biomaterials. 2014; 35(21): 5565-5571, doi: 10.1016/j.biomaterials.2014.03.018, indexed in Pubmed: 24731715.

2. Fong ELS, Chan CK, Goodman SB. Stem cell homing in musculoskeletal injury. Biomaterials. 2011; 32(2): 395-409, doi: 10.1016/j.biomaterials.2010.08.101, indexed in Pubmed: 20933277.

3. Sha Y, Lv Y, Xu Z, et al. MGF E peptide pretreatment improves the proliferation and osteogenic differentiation of BMSCs via MEK-ERK1/2 and PI3K-Akt pathway under severe hypoxia. Life Sci. 2017; 189: 52-62, doi: 10.1016/j. lfs.2017.09.017, indexed in Pubmed: 28927682.

4. Wu J, Zhang W, Ran Q, et al. The differentiation balance of bone marrow mesenchymal stem cells is crucial to hematopoiesis. Stem Cells Int. 2018; 2018: 1540148, doi: 10.1155/2018/1540148, indexed in Pubmed: 29765406.

5. Kawakami Y, Ii M, Matsumoto T, et al. SDF-1/CXCR4 axis in Tie2-lineage cells including endothelial progenitor cells contributes to bone fracture healing. J Bone Miner Res. 2015; 30(1): 95-105, doi: 10.1002/jbmr.2318, indexed in Pubmed: 25130304.

6. Peyvandi AA, Roozbahany NA, Peyvandi H, et al. Critical role of SDF-1/CXCR4 signaling pathway in stem cell homing in the deafened rat cochlea after acoustic trauma. Neural Regen Res. 2018; 13(1): 154-160, doi: 10.4103/1673-5374.224382, indexed in Pubmed: 29451220.

7. Zhu W, Liang G, Huang Z, et al. Conditional inactivation of the CXCR4 receptor in osteoprecursors reduces postnatal bone formation due to impaired osteoblast development. J Biol Chem. 2011; 286(30): 26794-26805, doi: 10.1074/jbc. M111.250985, indexed in Pubmed: 21636574.

8. Herberg S, Kondrikova G, Hussein KA, et al. Mesenchymal stem cell expression of stromal cell-derived factor- $1 \beta$ augments bone formation in a model of local regenerative therapy. J Orthop Res. 2015; 33(2): 174-184, doi: 10.1002/ jor.22749, indexed in Pubmed: 25351363.

9. Toupadakis CA, Wong A, Genetos DC, et al. Long-term administration of AMD3100, an antagonist of SDF-1/CXCR4 signaling, alters fracture repair. J Orthop Res. 2012; 30(11): 1853-1859, doi: 10.1002/jor.22145, indexed in Pubmed: 22592891.

10. Liles W, Broxmeyer H, Rodger E, et al. Mobilization of hematopoietic progenitor cells in healthy volunteers by AMD3100, a CXCR4 antagonist. Blood. 2003; 102(8): 2728-2730, doi: 10.1182/blood-2003-02-0663, indexed in Pubmed: 12855591.

11. Joshi R, Kulkarni YA, Wairkar S. Pharmacokinetic, pharmacodynamic and formulations aspects of Naringenin: An update. Life Sci. 2018; 215: 43-56, doi: 10.1016/j.lfs.2018.10.066, indexed in Pubmed: 30391464.

12. Cavia-Saiz M, Busto MD, Pilar-Izquierdo MC, et al. Antioxidant properties, radical scavenging activity and biomolecule protection capacity of flavonoid naringenin and its glycoside naringin: a comparative study. J Sci Food Agric. 2010; 90(7): 1238-1244, doi: 10.1002/jsfa.3959, indexed in Pubmed: 20394007.

13. Li YR, Chen DY, Chu CL, et al. Naringenin inhibits dendritic cell maturation and has therapeutic effects in a murine model of collagen-induced arthritis. J Nutr Biochem. 2015; 26(12): 1467-1478, doi: 10.1016/j.jnutbio.2015.07.016, indexed in Pubmed: 26350255. 
14. Pinho-Ribeiro FA, Zarpelon AC, Fattori V, et al. Naringenin reduces inflammatory pain in mice. Neuropharmacology. 2016; 105: 508-519, doi: 10.1016/j.neuropharm.2016.02.019, indexed in Pubmed: 26907804.

15. Yi LT, Li CF, Zhan X, et al. Involvement of monoaminergic system in the antidepressant-like effect of the flavonoid naringenin in mice. Prog Neuropsychopharmacol Biol Psychiatry. 2010; 34(7): 1223-1228, doi: 10.1016/j.pnpbp.2010.06.024, indexed in Pubmed: 20603175.

16. Kaczmarczyk-Sedlak I, Wojnar W, Zych M, et al. Effect of dietary flavonoid naringenin on bones in rats with ovariectomy-induced osteoporosis. Acta Pol Pharm. 2016; 73(4): 1073-1081, indexed in Pubmed: 29648734.

17. Swarnkar G, Sharan K, Siddiqui JA, et al. A naturally occurring naringenin derivative exerts potent bone anabolic effects by mimicking oestrogen action on osteoblasts. $\mathrm{Br}$ J Pharmacol. 2012; 165(5): 1526-1542, doi: 10.1111/j.14765381.2011.01637.x, indexed in Pubmed: 21864313.

18. Wang X, Zhen L, Zhang Ge, et al. Osteogenic effects of flavonoid aglycones from an osteoprotective fraction of Drynaria fortunei-an in vitro efficacy study. Phytomedicine. 2011; 18(10): 868-872, doi: 10.1016/j.phymed.2011.01.022, indexed in Pubmed: 21377852.

19. Wang $\mathrm{W}, \mathrm{Wu} \mathrm{C}$, Tian Bo, et al. The inhibition of RANKL-induced osteoclastogenesis through the suppression of p38 signaling pathway by naringenin and attenuation of titanium-particle-induced osteolysis. Int J Mol Sci. 2014; 15(12): 21913-21934, doi: 10.3390/ijms151221913, indexed in Pubmed: 25464380.

20. Wang W, Li M, Luo M, et al. Naringenin inhibits osteoclastogenesis through modulation of helper T cells-secreted IL4. J Cell Biochem. 2018; 119(2): 2084-2093, doi: 10.1002/ jcb.26370, indexed in Pubmed: 28834554.

21. Azizi SA, Stokes D, Augelli BJ, et al. Engraftment and migration of human bone marrow stromal cells implanted in the brains of albino rats-similarities to astrocyte grafts. Proc Natl Acad Sci U S A. 1998; 95(7): 3908-3913, doi: 10.1073/ pnas.95.7.3908, indexed in Pubmed: 9520466.

22. Yin Y, Li F, Li S, et al. TLR4 influences hepatitis B virus related hepatocellular carcinoma by regulating the Wnt $/ \beta$-catenin pathway. Cell Physiol Biochem. 2017; 42(2): 469-479, doi: 10.1159/000477594, indexed in Pubmed: 28578348.

23. Zhang $\mathrm{X}, \mathrm{Du} \mathrm{Yu}$, Ling J, et al. Dickkopf-related protein 3 negatively regulates the osteogenic differentiation of rat dental follicle cells. Mol Med Rep. 2017; 15(4): 1673-1681, doi: 10.3892/mmr.2017.6165, indexed in Pubmed: 28259940.

24. Fang T, Wang Y, Ma Y, et al. A rapid LC/MS/MS quantitation assay for naringin and its two metabolites in rats plasma. J Pharm Biomed Anal. 2006; 40(2): 454-459, doi: 10.1016/j. jpba.2005.07.031, indexed in Pubmed: 16406442.

25. Hübel K, Liles W, Broxmeyer H, et al. Leukocytosis and mobilization of $\mathrm{CD} 34+$ hematopoietic progenitor cells by AMD3100, a CXCR4 antagonist. Supportive Cancer Therapy. 2004; 1(3): 165-172, doi: 10.3816/sct.2004.n.008, indexed in Pubmed: 18628138.

26. Toupadakis CA, Wong A, Genetos DC, et al. Long-term administration of AMD3100, an antagonist of SDF-1/CXCR4 signaling, alters fracture repair. J Orthop Res. 2012; 30(11): 1853-1859, doi: 10.1002/jor.22145, indexed in Pubmed: 22592891.
27. Kitaori T, Ito H, Schwarz EM, et al. Stromal cell-derived factor $1 / \mathrm{CXCR} 4$ signaling is critical for the recruitment of mesenchymal stem cells to the fracture site during skeletal repair in a mouse model. Arthritis Rheum. 2009; 60(3): 813-823, doi: 10.1002/art.24330, indexed in Pubmed: 19248097.

28. Ming LG, Ge BF, Wang MG, et al. Comparison between 8-prenylnarigenin and narigenin concerning their activities on promotion of rat bone marrow stromal cells' osteogenic differentiation in vitro. Cell Prolif. 2012; 45(6): 508-515, doi: 10.1111/j.13652184.2012.00844.x, indexed in Pubmed: 23106298.

29. Yu GY, Zheng GZ, Chang Bo, et al. Naringin stimulates osteogenic differentiation of rat bone marrow stromal cells via activation of the notch signaling pathway. Stem Cells Int. 2016; 2016: 7130653, doi: 10.1155/2016/7130653, indexed in Pubmed: 27069482.

30. Tamamura H, Kuroda M, Masuda M, et al. A comparative study of the solution structures of tachyplesin I and a novel anti-HIV synthetic peptide, T22 ([Tyr5,12, Lys7]-polyphemusin II), determined by nuclear magnetic resonance. Biochim Biophys Acta. 1993; 1163(2): 209-216, doi: 10.1016/01674838(93)90183-r, indexed in Pubmed: 8490053.

31. Nakashima H, Masuda M, Murakami T, et al. Anti-human immunodeficiency virus activity of a novel synthetic peptide, T22 ([Tyr-5,12, Lys-7]polyphemusin II): a possible inhibitor of virus-cell fusion. Antimicrob Agents Chemother. 1992; 36(6): 1249-1255, doi: 10.1128/aac.36.6.1249, indexed in Pubmed: 1384424 .

32. Kitaori T, Ito H, Schwarz EM, et al. Stromal cell-derived factor $1 / \mathrm{CXCR} 4$ signaling is critical for the recruitment of mesenchymal stem cells to the fracture site during skeletal repair in a mouse model. Arthritis Rheum. 2009; 60(3): 813-823, doi: 10.1002/art.24330, indexed in Pubmed: 19248097.

33. Yang F, Xue F, Guan J, et al. Stromal-cell-derived factor (SDF) 1-alpha overexpression promotes bone regeneration by osteogenesis and angiogenesis in osteonecrosis of the femoral head. Cell Physiol Biochem. 2018; 46(6): 2561-2575, doi: 10.1159/000489684, indexed in Pubmed: 29758548.

34. Wang S, Zhou C, Zheng H, et al. Chondrogenic progenitor cells promote vascular endothelial growth factor expression through stromal-derived factor-1. Osteoarthritis Cartilage. 2017; 25(5): 742-749, doi: 10.1016/j.joca.2016.10.017, indexed in Pubmed: 27989872.

35. Chen HT, Tsou HK, Hsu CJ, et al. Stromal cell-derived factor-1/CXCR4 promotes IL-6 production in human synovial fibroblasts. J Cell Biochem. 2011; 112(4): 1219-1227, doi: 10.1002/jcb.23043, indexed in Pubmed: 21312239.

36. Wang H, Li C, Li J, et al. Naringin enhances osteogenic differentiation through the activation of ERK signaling in human bone marrow mesenchymal stem cells. Iran J Basic Med Sci. 2017; 20(4): 408-414, doi: 10.22038/IJBMS.2017.8582, indexed in Pubmed: 28804610.

37. Wu JB, Fong YC, Tsai HY, et al. Naringin-induced bone morphogenetic protein-2 expression via PI3K, Akt, c-Fos/cJun and AP-1 pathway in osteoblasts. Eur J Pharmacol. 2008; 588(2-3): 333-341, doi: 10.1016/j.ejphar.2008.04.030, indexed in Pubmed: 18495116.

38. Hernández-Aquino E, Muriel P. Beneficial effects of naringenin in liver diseases: Molecular mechanisms. World J Gastroenterol. 2018; 24(16): 1679-1707, doi: 10.3748/wjg.v24. i16.1679, indexed in Pubmed: 29713125.

Submitted: 14 October, 2020 Accepted after reviews: 3 March, 2021 Available as AoP: 11 March, 2021 